\title{
The Effect of Aspect Ratio and Deformability on Nanoparticle Extravasation through Nanopores
}

\author{
Farrell R. Kersey ${ }^{\dagger, \ddagger}$, Timothy J. Merke ${ }^{\dagger, \ddagger}$, Jillian L. Perry ${ }^{\dagger, \ddagger}$, Mary E. Napier ${ }^{\dagger, \ddagger}$, and Joseph \\ M. DeSimone ${ }^{\star}, \dagger, \neq, \S, \perp, \|, \#$ \\ †Department of Chemistry, University of North Carolina at Chapel Hill, Chapel Hill, NC 27599 \\ ‡Lineberger Comprehensive Cancer Center, University of North Carolina at Chapel Hill, Chapel \\ Hill, NC 27599 \\ $\S$ Carolina Center of Cancer Nanotechnology Excellence, University of North Carolina at Chapel \\ Hill, Chapel Hill, NC 27599 \\ ${ }^{\perp}$ Institute for Nanomedicine, University of North Carolina at Chapel Hill, Chapel Hill, NC 27599 \\ "Institute for Materials Science, University of North Carolina at Chapel Hill, Chapel Hill, NC 27599 \\ \#Department of Chemical and Biomolecular Engineering, North Carolina State University, \\ Raleigh, NC 27695
}

\begin{abstract}
We describe the fabrication of filamentous hydrogel nanoparticles using a unique soft lithography based particle molding process referred to as PRINT (Particle Replication in Non-wetting Templates). The nanoparticles possess a constant width of $80 \mathrm{~nm}$, and we varied their lengths ranging from $180 \mathrm{~nm}$ to $5000 \mathrm{~nm}$. In addition to varying the aspect ratio of the particles, the deformability of the particles was tuned by varying the cross-link density within the particle matrix. Size characteristics such as hydrodynamic diameter and persistence length of the particles were analyzed using dynamic light scattering and electron microscopy techniques, respectively, while particle deformability was assessed by atomic force microscopy. Additionally, the ability of the particles to pass through membranes containing $0.2 \mu \mathrm{m}$ pores was assessed by means of a simple filtration technique, and particle recovery was determined using fluorescence spectroscopy. The results show that particle recovery is mostly independent of aspect ratio at all cross-linker concentrations utilized, with the exception of $96 \mathrm{wt} \%$ PEG diacrylate $80 \times 5000 \mathrm{~nm}$ particles, which showed the lowest percent recovery.
\end{abstract}

\section{Keywords}

PRINT; soft lithography; high-aspect-ratio nanoparticles; filtration; extravasation

\section{Introduction}

In the growing field of nanomedicine, a number of nanoparticle properties have been shown to play a significant role in biodistribution, circulation, and cellular uptake. Such properties include, but are not limited to, particle geometry, size, charge, surface functionality, and

\footnotetext{
Corresponding author desimone@unc.edu.

Supporting Information Available. A table of particle $D_{\mathrm{h}}$ values before and after filtration, SEM images of $96 \mathrm{wt} \%$ PEGDA filamentous nanoparticles before and after filtration, and representative SEM images of $80 \times 5000 \mathrm{~nm}$ nanoparticles used for persistence length analysis. This material is available free of charge via the Internet at http://pubs.acs.org.
} 
modulus. ${ }^{1-6}$ Of these properties, modulus, being one of the least studied properties among nanoparticles, can notably affect these biological processes. For example, soft spherical acrylamide-based nanogels were recently reported to deform and pass through membrane pores several times smaller than their hydrodynamic diameter under physiological pressures. ${ }^{7}$ Also, varying the moduli of similar acrylamide-based nanoparticles has been shown to affect their rate of uptake by macrophages in vitro. ${ }^{8}$ Additionally, non-spherical hydrogel-based microparticles with low moduli have been reported to pass through narrow microfluidic channels. ${ }^{9}$ This behavior was also observed with hydrogel-based red blood cell mimics, which were also studied in vivo and showed prolonged circulation of up to five days. ${ }^{10}$

Among the various nanoparticle geometries available for study, filamentous or high aspect ratio particles represent a particularly interesting geometry that is difficult to access in a systematic and controllable fashion. Such shapes are found repeatedly throughout nature in the form of bacteria such as E. coli and the filoviridae family of viruses, ${ }^{11}$ leading to the theory that the filamentous shape of these pathogens is the result of adaption to external conditions and selective forces. ${ }^{12}$ Recent investigations show promise for filamentous nanoparticles to act as efficient therapeutic and imaging agents, capable of encapsulating and delivering cargos such as Paclitaxel and siRNA. ${ }^{13-16}$ In addition to this, these particles have shown a higher propensity for avoiding macrophage uptake in vitro than their spherical counterparts. $2,6,17-19$ Carbon nanotubes and metallic nanorods and nanowires fall into the class of filamentous nanoparticles; ${ }^{20}$ however, their rigid structure and chemical compositions often make them incompatible with biological systems unless pegylated to avoid rapid clearance. ${ }^{21,22}$ It has been hypothesized that soft filamentous nanoparticles have the potential to be taken up by the enhanced permeation and retention (EPR) effect, and, due to their geometry, be less likely to escape tumor tissue. ${ }^{13}$ Also, as previously mentioned, their enhanced ability to avoid macrophage clearance makes them ideal for longer circulation.

Fabrication of filamentous nanoparticles has been previously achieved using bottom-up and top-down approaches. Most bottom-up approaches have involved creating wormlike micelles or "filomicelles" through morphological changes to polymer vesicles. ${ }^{23,24}$ Filomicelles created from diblock copolymers of poly(ethylene glycol) with blocks of poly(lactic acid) or polycaprolactone are both biocompatible and are capable of undergoing hydrolysis in the process of delivering therapeutic cargos. ${ }^{25,26}$ The stiffness of filomicelles can be tuned by the use of diblock copolymers over a range of molecular weights. ${ }^{14,27}$ Stiff filomicelles have also been obtained using a poly(ethylethylene) or poly(butadiene) block segment which can be cross-linked to form a rigid core. ${ }^{15,27,28}$ Dimensions of filomicelles range from $\sim 10-60 \mathrm{~nm}$ in diameter and up to $18 \mu \mathrm{m}$ in length, and they have been observed to circulate for several days, hypothesized to be a result of their ability to avoid macrophage uptake and clearance. Although these results are impressive, the dynamic nature of the particles and the lack of control over particle length could represent possible disadvantages, such as shearing apart under blood flow or during extrusion through the fenestrated capillaries of organs.

Particles of various shapes and sizes, including filamentous nanoparticles, have also been fabricated using the polystyrene bead stretching method first described by Ho et al. ${ }^{29}$ Mitragotri and coworkers have used this method extensively for creating filamentous nanoparticles to study macrophage uptake properties $2,6,17,18$ and recently reported that polymeric nanoneedles are effective at delivering siRNA to cells. ${ }^{30}$ Monodisperse particles are not necessarily achievable by means of this method since the final particle size is dependent on the starting dispersity of the polystyrene beads. Additionally, the filamentous 
nanoparticles produced by this method typically have larger widths $(\sim 200 \mathrm{~nm})$, and the material used is not biocompatible or biodegradable.

Bilayer nanoimprint lithography (B-NIL) has been used to fabricate long filamentous particles, ${ }^{31}$ as well as shorter particles in the form of bullets and rods, ${ }^{32}$ using epoxy-based SU-8 photoresist. The B-NIL method allows for the fabrication of monodisperse particles which can be easily removed and collected from the surface of a sacrificial layer of soluble polymer. The dimensions of filamentous particles created using this method have ranged from $300 \mathrm{~nm}$ to $6 \mu \mathrm{m}$ in length with a constant width of $80 \mathrm{~nm}$ in diameter. While the longer filamentous particles show some degree of localized flexibility in their structure, they otherwise appear quite rigid, and control over particle stiffness has yet to be demonstrated.

Recent publications from our group have detailed the use of the technique known as Particle Replication in Non-wetting Templates (PRINT). ${ }^{3-5,33-36}$ This versatile method allows for the fabrication of particles with variations in shape, size, surface charge, stiffness, and chemical composition. Using this technique it has been shown that particles can be prepared from several biocompatible and biodegrable materials such as PEG, ${ }^{33}$ proteins, ${ }^{37}$ and poly(lactic-co-glycolic acid). ${ }^{38}$ PRINT also allows for tunability of particle stiffness. In the case of UV curable systems such as acrylates, stiffness can be tuned by variations in crosslinker concentration and length, as we have previously shown with hydrogel-based red blood cell mimics. ${ }^{10}$ An additional advantage of PRINT is the ability to scale up particle production by means of reel-to-reel systems.

Studies to date have only provided a glimpse at how particle deformability might influence in vitro and in vivo properties such as uptake and biodistribution, especially in regards to unique shapes such as filaments. Therefore, determining the deformation properties of filamentous nanoparticles is an essential first step before moving on to in vivo models. We discuss here the fabrication of monodisperse, filamentous PEG-based hydrogel nanoparticles with a diameter of $80 \mathrm{~nm}$ and lengths ranging from $180 \mathrm{~nm}$ to $5000 \mathrm{~nm}$ using the PRINT method. Particle stiffness was controlled by varying the cross-linker concentration within the PEG-based particle matrix, and the effect of cross-linker concentration on particle size was analyzed using dynamic light scattering (DLS). As previously mentioned, microfluidics is often used to look at the extent of particle deformability for microparticles with widths greater than that of the device channels. However, the narrowest channels in most microfluidic devices are on the order of several microns, making them impractical for deformation studies with filamentous nanoparticles. In order to assess the extravasation properties of the filamentous nanoparticles in this study, an assay was created in which the particles were filtered through a membrane with pore sizes greater in width than the $80 \mathrm{~nm}$ diameter of the particles. The extent of particle recovery as a function of cross-linker concentration was assessed by measuring the percentage of recovered particles that could be accrued upon a single pass through a 0.2 micron PTFE filter as determined by fluorescence spectroscopy of fluorophore labeled particles. The extent of particle deformation as a function of cross-linker concentration was also described using a previously reported surface spreading assay using atomic force microscopy (AFM). ${ }^{39}$ Additionally, particle flexibility was assessed by SEM, which allowed for the determination of an equilibrated, 2D particle persistence length.

\section{Experimental Section}

\section{Materials}

Poly(ethylene glycol) diacrylate $\left(\mathrm{M}_{\mathrm{n}} 700\right)$ was purchased from Sigma-Aldrich and passed through a column of adsorption alumina (Fisher Scientific) to remove inhibitor prior to use. Tetraethylene glycol monoacrylate $\left(\mathrm{HP}_{4} \mathrm{~A}\right)$ was synthesized as previously described. ${ }^{40} 2,2$ - 
Diethoxyacetophenone (DEAP, $>95 \%$, Acros), methacryloxyethyl thiocarbamoyl rhodamine B (PolyFluor570, Polysciences, Inc.), polyvinyl alcohol (PVOH, MW 2 kDa, Acros), Luvitec (MW $64 \mathrm{kDa}, \mathrm{BASF}$ ), and methanol (99.9\% anhydrous, Acros) were used as received. Thermo Scientific HyperClean PTFE syringe filters (4 mm membrane, $0.2 \mu \mathrm{m}$ pore size) with medical grade polypropylene housing were obtained from Fisher Scientific. PRINT molds, $80 \times 180 \mathrm{~nm}, 80 \times 320 \mathrm{~nm}, 80 \times 2000 \mathrm{~nm}$, and $80 \times 5000 \mathrm{~nm}$ were obtained from Liquidia Technologies.

\section{PRINT Particle Fabrication}

The PRINT method for particle fabrication has been previously described in detail. Preparticle solutions were prepared by first combining $2 \mathrm{mg}$ PolyFluor570 and $2 \mathrm{mg}$ DEAP. Depending upon final particle composition, $2 \mathrm{mg}, 10 \mathrm{mg}$, $50 \mathrm{mg}$, or $96 \mathrm{mg}$ PEGDA was then added. With the exception of $96 \mathrm{mg}$ PEGDA, $\mathrm{HP}_{4} \mathrm{~A}$ was subsequently added to bring the total mass to $100 \mathrm{mg}$. The organics were briefly sonicated and diluted with the necessary volumes of anhydrous methanol to prepare $2 \%$ and $2.5 \% \mathrm{w} / \mathrm{w}$ solutions. Solutions were then filtered through a $0.2 \mu \mathrm{m}$ PTFE $25 \mathrm{~mm}$ filter (Fisher Scientific) to remove particulates.

For hydrogel particles containing $50 \mathrm{wt} \%$ and $96 \mathrm{wt} \%$ PEGDA, fabrication was performed as follows: a $2 \% \mathrm{w} / \mathrm{w}$ solids pre-particle solution in methanol $(2.5 \% \mathrm{w} / \mathrm{w}$ for $80 \times 180 \mathrm{~nm}$ particles) was first prepared. A sheet of polyethylene terephthalate (PET) was coated with the desired preparticle solution composition using $350 \mathrm{~mL}$ preparticle solution and a \#5 Mayer rod (R.D. Specialties). The solvent was evaporated by briefly exposing the film to a heat gun. The film was then laminated to the patterned side of the PRINT mold at $50 \mathrm{psi}$, followed by delaminating under the same conditions. The filled mold was subsequently laminated against a sheet of PET and slowly passed through a heated nip (ChemInstruments Hot Roll Laminator) at $300^{\circ} \mathrm{F}$ and $80 \mathrm{psi}$. The mold-PET sandwich was then allowed to cool to room temperature before curing. Curing was performed in a nitrogen-purged UV oven $\left(\lambda=365 \mathrm{~nm}\right.$, power $\left.\geq 20 \mathrm{~mW} / \mathrm{cm}^{2}\right)$ for three minutes. The particles were then harvested from the PET sheet with a small volume $(\sim 1 \mathrm{~mL})$ of deionized water containing $0.2 \mathrm{wt} \% \mathrm{PVOH}$ using a cell scraper (Fisher Scientific).

For hydrogel particles containing $2 \mathrm{wt} \%$ and $10 \mathrm{wt} \%$ PEGDA, film preparation was performed in the same manner as described above. The film was then laminated to the patterned side of the PRINT mold 3-5 times at 50 psi before delaminating. The particles were then cured in the same manner as the mold-PET sandwich previously described. Cured molds were laminated to a water-soluble polymer film (Plasdone or PVOH) on a PET sheet at $300{ }^{\circ} \mathrm{F}$ and $80 \mathrm{psi}$ and allowed to cool to room temperature. The mold was then peeled away leaving the particles embedded in the polymer layer. The polymer layer containing the particles was then harvested into a bead of deionized water $(\sim 1 \mathrm{~mL})$ using a custom-made bead harvester.

All particle solutions were concentrated via tangential flow filtration with a MicroKros hollow fiber filter (500 kDa MWCO, Spectrum Laboratories, Inc.) to remove residual sol fraction and unreacted dye. Concentrated particle solutions were then backfilled with water between washes. Finally, concentrated particle solutions were backfilled no less than two times with an aqueous stabilizer solution containing $0.1 \mathrm{wt} \% \mathrm{PVOH}$ and $0.1 \mathrm{wt} \%$ Luvitec and reconcentrated.

\section{Particle Characterization}

Stock particle concentrations were determined by thermogravimetric analysis (TGA) using a TA Instruments Q5000 TGA. Briefly, $20 \mu \mathrm{L}$ of stock solution was pipetted into a tared aluminum sample pan. The sample was heated at $50^{\circ} \mathrm{C} / \mathrm{min}$ to $130^{\circ} \mathrm{C}$, followed by a 10 
minute isotherm. The sample was then cooled at $50{ }^{\circ} \mathrm{C} / \mathrm{min}$ to $30{ }^{\circ} \mathrm{C}$, followed by a 5 minute isotherm. TGA was also performed on a $20 \mu \mathrm{L}$ aliquot of supernatant from a centrifuged sample of each stock solution to account for the mass of stabilizer in each sample. The concentration of the stabilizer solution was subtracted from the concentration of the stock particle solution to determine the actual particle concentration.

Particles were visualized by scanning electron microscopy (SEM) using a Hitachi S-4700 SEM. Particle arrays or harvested particles on glass were coated with 1.5 to $2 \mathrm{~nm}$ of goldpalladium alloy using a Cressington 108 auto sputter coater. Images were obtained at an accelerating voltage of $2.0 \mathrm{kV}$. Fluorescence microscopy was performed on $80 \times 2000 \mathrm{~nm}$ and $80 \times 5000 \mathrm{~nm}$ particles using an Olympus $\mathrm{I} \times 81$ Microscope with a $100 \times$ oil objective. For size characterization in solution, dynamic light scattering (DLS) measurements were obtained using a Nano ZS Zetasizer (Malvern Instruments) to determine effective hydrodynamic diameter $\left(D_{\mathrm{h}}\right)$. The parameters used for DLS measurements were a backscattering angle $\Theta=173^{\circ}$ and laser wavelength $\lambda=633 \mathrm{~nm}$. This angle provides greater sensitivity and reduces artifacts associated with variations in solution concentration, while the laser wavelength was chosen to inhibit absorption by the rhodamine monomer within the particle compositions. Size measurements were also performed in the default instrument range of $0.6 \mathrm{~nm}$ to $6 \mu \mathrm{m}$.

\section{AFM Studies}

$80 \times 180 \mathrm{~nm}$ nanoparticles of varying PEGDA concentrations were characterized by atomic force microscopy using an Asylum Research MFP-3D atomic force microscope. Height, phase, and amplitude images were acquired in air in tapping mode with a silicon cantilever (Budget Senors TAP300A1-G, $\omega=300 \mathrm{kHz}, k_{\mathrm{c}}=40 \mathrm{~N} / \mathrm{m}$ ) at a scan rate of $1 \mathrm{~Hz}$. Samples for imaging were prepared by pipetting a $30 \mu \mathrm{L}$ aliquot of $0.17 \mathrm{mg} / \mathrm{mL}$ particle solution onto a clean $1 \mathrm{~cm}^{2}$ glass slide. The glass slides were then heated at $80^{\circ} \mathrm{C}$ for several minutes to allow for evaporation of water.

\section{Persistence Length Determination}

Persistence lengths were determined from SEM images of 10, 50, and $96 \mathrm{wt} \%$ PEGDA $80 \times$ $5000 \mathrm{~nm}$ particles using the 2D Single Molecule software package provided by Prof. Sergiy Minko (http://people.clarkson.edu/ sminko).$^{41}$ Briefly, the filamentous nanoparticles' coordinates were recorded by dragging a cursor along the zoomed contours of clearly isolated particles. The recorded coordinates were used to estimate the experimental values of particle contour length, $L_{\mathrm{C}}$, and persistence length, $L_{\mathrm{P}}$.

\section{Particle Filtration}

For particle filtration experiments $500 \mu \mathrm{L}$ of particle solution with a concentration of 0.17 $\mathrm{mg} / \mathrm{mL}$ was drawn up into a $5 \mathrm{~mL}$ syringe. The syringe containing the solution was then fitted with a pre-wetted $4 \mathrm{~mm}$ HyperClean PTFE filter with pore sizes of $0.2 \mu \mathrm{m}$. Based on pore-size ratings provided by the manufacturer, $0.2 \mu \mathrm{m}$ defines the maximum diameter of a channel through the filter medium and represents a nominal value. The syringe plunger was pulled back leaving an air gap between the solution and the plunger, and the particle solution was filtered by applying pressure manually to the plunger, discarding the first 1-2 droplets to rule out dilution due to pre-wetting of the filter. The pressure across the filter was monitored using a pressure gauge and did not exceed $\sim 40 \mathrm{psi}$ (pressure drop $=25.3 \mathrm{psi}$ ). In some cases where clogging occurred, device failure was noted by a sudden drop in pressure or dislodging of the filter from the device housing. Experiments where device failure occurred were not included in the final data analysis. Filtrations were performed in triplicate, and the filtered particle solution was collected in full unless clogging occurred during the course of filtration. Fluorescence intensities of particle solutions before and after filtration were 
acquired using a SpectraMax M5 plate reader (Molecular Devices) with parameters set to the experimentally determined excitation and emission wavelengths of PolyFluor570 ( $\lambda_{\mathrm{ex}}=$ $\left.548 \mathrm{~nm}, \lambda_{\mathrm{em}}=580 \mathrm{~nm}\right)$. To determine the $\%$ recovered fluorescence solutions of particles before and after filtration were pipetted into a 96-well plate at constant volume. Deionized water was used as a blank. Measured fluorescence intensities were corrected by subtracting out the fluorescence intensity of the blank. Percent recovered fluorescence was then determined by dividing the filtered particle solution fluorescence intensity by the pre-filtered particle solution fluorescence intensity.

\section{Results and Discussion}

\section{Fabrication of Filamentous Hydrogel-based Nanoparticles using PRINT}

A series of nanoparticles with a constant diameter of $80 \mathrm{~nm}$ and lengths ranging from 180 $\mathrm{nm}$ to $5000 \mathrm{~nm}$ were successfully fabricated using PRINT. Particle stiffness was controlled by varying PEGDA concentrations between $2 \mathrm{wt} \%$ and $96 \mathrm{wt} \%$ within the particle composition. The remainder of the composition was comprised of photoinitiator ( $2 \mathrm{wt} \%)$, PolyFluor570 dye ( $2 \mathrm{wt} \%)$, and the hydrophilic monomer $\mathrm{HP}_{4} \mathrm{~A}$. Particles were fabricated in an ordered array, as shown in the SEM images in Figure 1, and the monodisperse nature of the particles can be clearly seen. The SEM image in Figure 2 shows $80 \times 5000 \mathrm{~nm}$ particles comprised of $96 \mathrm{wt} \%$ PEGDA which have partially detached from the PET sheet on which they were fabricated. Although these particles are the stiffest of the compositions studied, they possess some degree of flexibility which is visible in the image. This flexibility can also be seen in fluorescence microscope images of the particles, shown in the inset of Figure 2.

\section{Dynamic Light Scattering of Filamentous Hydrogel-based Nanoparticles}

Dynamic light scattering (DLS) is most commonly used to characterize the hydrodynamic size of molecules such as polymers and proteins, or larger structures such as spherical nanoand microparticles. In some instances DLS can aid in determining size properties of nonspherical particles and has been used to successfully characterize rod-like particles and worm-like micelles. ${ }^{14,42,43}$ DLS was used here to determine the effective hydrodynamic diameter $\left(D_{\mathrm{h}}\right)$ of filamentous nanoparticles as a function of aspect ratio (AR) and crosslinker concentration, the results of which are shown in Figure 3. It should be noted, however, that $D_{\mathrm{h}}$ here is not indicative of a quantitative length scale and only acts to compare relative trends.

For $80 \times 180 \mathrm{~nm}$ particles a gradual decrease in $D_{\mathrm{h}}$ at $25^{\circ} \mathrm{C}$ was observed as a function of PEGDA concentration, where sizes ranged from $272 \pm 78 \mathrm{~nm}$ at $2 \mathrm{wt} \%$ PEGDA to $176 \pm 6$ $\mathrm{nm}$ at $96 \mathrm{wt} \%$ PEGDA. A similar trend was also observed at $37^{\circ} \mathrm{C}$, indicating particle size to be independent of temperature at this length scale. The decrease in $D_{\mathrm{h}}$ with increasing PEGDA concentration suggests that as the extent of cross-linking decreases swelling of the particles increases, which has been previously observed for spherical nanogels. ${ }^{44}$ Since $80 \times$ $180 \mathrm{~nm}$ particles $(\mathrm{AR}=2.25)$ are only slightly larger in aspect ratio than spherical nanoparticles (AR $=1)$, the decrease in $D_{\mathrm{h}}$ with increasing PEGDA concentration is not unexpected.

In the case of $80 \times 320 \mathrm{~nm}$ particles $(\mathrm{AR}=4)$ no notable trend was observed as a function of PEGDA concentration. $D_{\mathrm{h}}$ values were found to range from $339 \pm 7 \mathrm{~nm}$ at $2 \mathrm{wt} \%$ PEGDA to $290 \pm 6 \mathrm{~nm}$ at $96 \mathrm{wt} \%$ PEGDA, and particles with intermediate concentration of PEGDA where observed to be of similar size. Since the shape of these particles falls between a spherical and rod-like confirmation, similar to a prolate spheroid, any trends associated with $D_{\mathrm{h}}$ values as a function of PEGDA concentration may not be distinguishable. Additionally, 
the $D_{\mathrm{h}}$ values measured for $80 \times 320 \mathrm{~nm}$ particles appear to be independent of temperature over the range of PEGDA concentrations studied.

The most surprising results were found for $80 \times 2000 \mathrm{~nm}(\mathrm{AR}=25)$ and $80 \times 5000 \mathrm{~nm}(\mathrm{AR}$ $=62.5$ ) particles when examined by DLS. At $96 \mathrm{wt} \%$ PEGDA $D_{h}$ values for $80 \times 2000 \mathrm{~nm}$ and $80 \times 5000 \mathrm{~nm}$ particles were $775 \pm 34 \mathrm{~nm}$ and $1469 \pm 40 \mathrm{~nm}$, respectively. This increase in $D_{h}$ values is directly correlated with the increased particle length and falls within the expected trend observed for $80 \times 180 \mathrm{~nm}$ and $80 \times 320 \mathrm{~nm}$ particles at $96 \mathrm{wt} \%$ PEGDA. Interestingly, $D_{h}$ was observed to decrease as a function of decreasing PEGDA concentration for these longer particles. For $80 \times 2000 \mathrm{~nm}$ particles $D_{h}$ values were $740 \pm 36$ $\mathrm{nm}, 340 \pm 10 \mathrm{~nm}$, and $258 \pm 28 \mathrm{~nm}$ at $50 \mathrm{wt} \%, 10 \mathrm{wt} \%$, and $2 \mathrm{wt} \%$ PEGDA, respectively. Additionally, decreasing values for $D_{h}$ of $80 \times 5000 \mathrm{~nm}$ particles were found to be $1070 \pm$ $82 \mathrm{~nm}, 292 \pm 8 \mathrm{~nm}$, and $261 \pm 36 \mathrm{~nm}$ at $50 \mathrm{wt} \%, 10 \mathrm{wt} \%$, and $2 \mathrm{wt} \%$ PEGDA, respectively. The $D_{h}$ values for $80 \times 2000 \mathrm{~nm}$ and $80 \times 5000 \mathrm{~nm}$ particles were also found to be a function of temperature, especially at the higher PEGDA concentrations (>50 wt $\%)$. At 37 ${ }^{\circ} \mathrm{C} D_{h}$ values for $80 \times 2000 \mathrm{~nm}$ and $80 \times 5000 \mathrm{~nm}$ particles composed of $96 \mathrm{wt} \%$ PEGDA were $519 \pm 51 \mathrm{~nm}$ and $685 \pm 121 \mathrm{~nm}$, respectively, which is a significant decrease compared to values at $25{ }^{\circ} \mathrm{C}$. A similar decrease in $D_{h}$ was also observed for particles of $50 \mathrm{wt} \%$ PEGDA. An unexpected result was observed for $80 \times 2000 \mathrm{~nm}$ and $80 \times 5000 \mathrm{~nm}$ particles containing < $10 \mathrm{wt} \%$ PEGDA, where $D_{h}$ values were measured to be in the same range as those found for $80 \times 320 \mathrm{~nm}$ particles. A possible explanation for this is that as PEGDA concentration decreases, the modulus of the particles decreases, as expected. This decrease in modulus leads to increased flexibility, which also appears to be a function of temperature. An increase in temperature resulting in increased flexibility may allow filamentous particles to sample a larger number of spatial configurations, which is analogous to that of an individual polymer chain in solution.

\section{Effect of PEGDA Concentration on Particle Deformability - AFM Studies}

It has been well established that the extent of cross-linking within a polymeric network plays an important role in the resulting mechanical properties of the subsequent bulk material. ${ }^{45}$ Most often a cross-linked system with a high mesh density will lead to materials with high moduli, while a decrease in mesh density, creating a loosely cross-linked network, will result in lower moduli. Modulus can be further tuned by varying the size of the cross-linking moiety. For example, an increase in the length of a cross-linker such as PEGDA can lead to a higher degree of porosity within a material, ultimately resulting in decreased modulus. In the studies presented here PEGDA was used as the particle cross-linker due its previous use in creating hydrogel-based materials with a number of tunable physicochemical properties such as modulus and mesh size, both of which affect deformability. ${ }^{46-49}$

In order to determine the extent of deformability of the particles studied here, $80 \times 180 \mathrm{~nm}$ particles over the range of PEGDA concentrations utilized in this study were dried onto glass slides and imaged in tapping mode using atomic force microscopy (AFM).

Representative three-dimensional height images of $2 \mathrm{wt} \%$ and $96 \mathrm{wt} \%$ PEGDA particles are shown in Figure 4. It can be seen qualitatively that $80 \times 180 \mathrm{~nm}$ particles composed of $96 \mathrm{wt}$ $\%$ PEGDA exhibit the least deformability, essentially retaining their shape after drying to the glass slide. As the PEGDA concentration decreases to $2 \mathrm{wt} \%$, however, the particles show an increased tendency to flatten across the glass substrate. The ability of the nanoparticles to spread out across the substrate as a function of decreasing PEGDA concentration is a direct observation of their capacity to deform. Additional qualitative analysis of particle deformation is shown in Figure 5, which shows particle height increasing and particle width decreasing as a function of PEGDA concentration. 


\section{Effect of PEGDA Concentration on Filamentous Nanoparticle Persistence Length}

The persistence length $L_{\mathrm{P}}$ of a filament is a measure of its inherent flexibility, and, as such, is directly proportional to the modulus of the filament as follows:

$$
L_{\mathrm{P}}=Y I / \mathrm{k}_{\mathrm{B}} T
$$

where $Y$ is the Young's modulus of the filament, $I$ is the area moment of inertia of the filament, and $\mathrm{k}_{\mathrm{B}}$ and $T$ represent Boltzmann's constant and temperature, respectively. ${ }^{50}$ Previous studies have often focused on the use of fluorescence microscopy to determine 3D persistence lengths in solution for structures such as biofilaments ${ }^{51-53}$ and filomicelles, ${ }^{27,28,54}$ which allows for the determination of persistence lengths based on monitoring particle fluctuations over time. However, newer approaches have provided a simpler method for determining persistence lengths of filamentous particles in an energetically equilibrated 2D-configuration, such as those imposed when using techniques such as AFM ${ }^{54-56}$ and electron microscopy. 57,58 In the case of filaments that are energetically equilibrated on a solid support, the persistence length calculated is essentially identical to that of the same filament in a dilute solution. ${ }^{55}$

To determine $L_{\mathrm{P}}$ values for our filamentous nanoparticles, SEM images were collected for $80 \times 5000 \mathrm{~nm}$ particles composed of 10, 50, and $96 \mathrm{wt} \%$ PEGDA. The high degree of surface spreading that occurs with $2 \mathrm{wt} \%$ PEGDA particles upon drying on a substrate resulted in poor particle resolution by SEM. Therefore, this particle composition was not included in the final analysis. The collected SEM images were analyzed using the software previously described in the Experimental Section, and values for $L_{\mathrm{C}}$ and $L_{\mathrm{P}}$ were obtained. The results of the analysis are summarized in Table 1.

We see from Table 1 that the obtained values for $L_{C}$ are on the order of the expected value of $5000 \mathrm{~nm}$. While not significantly different when accounting for standard deviation, there is a noted increasing trend with decreasing PEGDA concentration for average $L_{\mathrm{C}}$. At $96 \mathrm{wt}$ $\%$ PEGDA the particle contour length was found to be $4.97 \mu \mathrm{m}$, while at 50 and $10 \mathrm{wt} \%$ PEGDA, $L_{\mathrm{C}}$ was $5.34 \mu \mathrm{m}$ and $5.40 \mu \mathrm{m}$, respectively. The $L_{\mathrm{C}}$ value for the $96 \mathrm{wt} \%$ PEGDA $80 \times 5000 \mathrm{~nm}$ particles suggests that little to no particle swelling occurs in aqueous solution due to the large amount of cross-linking, and the particle morphology does not significantly change upon deposition and drying to a solid substrate, as was done to collect the SEM images described. This behavior is corroborated by the AFM studies described earlier, where $96 \mathrm{wt} \%$ PEGDA $80 \times 180 \mathrm{~nm}$ particles showed no structural change after drying on a substrate. The increased $L_{\mathrm{C}}$ values determined for 10 and $50 \mathrm{wt} \%$ PEGDA particles, however, can be accounted for through particle swelling. The lower degrees of cross-linking within the hydrophilic materials allow for notable hydration as observed by DLS, where increases in $D_{\mathrm{h}}$ for $80 \times 180 \mathrm{~nm}$ particles where found at lower PEGDA concentrations ( $\leq$ $10 \mathrm{wt} \%$ ). This effect is also observed when particles with low cross-linking are imaged by AFM after drying to solid substrates, whereby the particles are seen to adhere and spread across the substrate, displaying a dramatic loss of morphological integrity.

Also shown in Table 1 are the values determined for persistence length $L_{\mathrm{P}}$ for the $80 \times 5000$ $\mathrm{nm}$ particle compositions in question. Surprisingly, $L_{\mathrm{P}}$ values calculated for 10 and $50 \mathrm{wt} \%$ PEGDA particles were similar in value $(1.61 \mu \mathrm{m}$ and $1.57 \mu \mathrm{m}$ for 10 and $50 \mathrm{wt} \%$ PEGDA, respectively). These particles can therefore be described as being in the range of flexible $\left(L_{\mathrm{C}}\right.$ $\left.\gg L_{\mathrm{P}}\right)$ to semi-flexible $\left(L_{\mathrm{C}} \approx L_{\mathrm{P}}\right)$, as opposed to rigid $\left(L_{\mathrm{C}} \ll L_{\mathrm{P}}\right)$ in nature. While these values are essentially the same, suggesting that the particles should have similar moduli and flexibility characteristics, $10 \mathrm{wt} \%$ PEGDA particles observed in the SEM images obtained often showed a higher degree of local bending and flexibility than $50 \mathrm{wt} \%$ PEGDA particles, 
which showed more global bending (Figure S2 in Supporting Information). Therefore, the persistence lengths in these two cases are more effectively described as apparent $L_{\mathrm{P}}$ since particle flexibility may be convoluted by artifacts such as drying/attachment mechanisms and substrate properties.

When $96 \mathrm{wt} \%$ PEGDA $80 \times 5000 \mathrm{~nm}$ particles were analyzed, an $L_{\mathrm{P}}$ value of $16.96 \mu \mathrm{m}$ was obtained. This is a 10 -fold increase in $L_{\mathrm{P}}$ compared to the 10 and $50 \mathrm{wt} \%$ PEGDA $80 \times$ $5000 \mathrm{~nm}$ particles previously discussed. The large increase in $L_{\mathrm{P}}$ seen here is not surprising, however, since the increase in cross-linking results in a material with increased modulus and decreased flexibility. The decreased particle flexibility can be seen in SEM images of the 96 wt $\%$ PEGDA $80 \times 5000 \mathrm{~nm}$ particles, where essentially all particles are observed to be straight, rigid rods (Figure S2 in Supporting Information), and the rigid rod description is backed up by the fact that $L_{\mathrm{C}}$ is much less than $L_{\mathrm{P}}$ for these particles, as shown in Table 1 .

\section{Filtration of Filamentous Nanoparticles}

The ability of filamentous nanoparticles to penetrate leaky vasculature is of vital importance for their potential role in oncology-based nanomedicine. It has been previously shown that tumor microvasculature can range from $200 \mathrm{~nm}$ to $1.2 \mu \mathrm{m}$, depending upon the tumor type and microenvironment in which it exists. ${ }^{59}$ Therefore, modulating the physical properties of filamentous nanoparticles may ultimately help in creating efficient particle uptake by tumors. This uptake mechanism may be dependent to some degree on the ability of the particles to deform under the stresses exerted during physiological processes. In order to determine the deformation characteristics of the filamentous nanoparticles reported here a simple filtration assay was developed. The assay involved passing a small volume of aqueous nanoparticle solution through a sterile syringe filter possessing a membrane with a pore-size rating of $0.2 \mu \mathrm{m}$. Commercially-available medical-grade syringe filters were chosen for particle filtrations since filters are often used for sterilization of nanoparticle formulations, which is a necessary step prior to clinical use. ${ }^{60,61}$

Following filtration, fluorescence intensities of pre- and post-filtration particle solutions were measured. The percent recovered fluorescence was calculated as the fluorescence intensity of the post-filtration solution divided by the fluorescence intensity of the prefiltration solution. Since purification of the particles leads to the removal of all unreacted dye from the particle solution, $\%$ recovered fluorescence can also be considered $\%$ particle recovery. Based on the findings of the filtration experiments, \% particle recovery is found to be mostly independent of PEGDA concentration at the smaller particle sizes of $80 \times 180 \mathrm{~nm}$ and $80 \times 320 \mathrm{~nm}$ (Figure 6), which fell in the range of $77 \%$ to $93 \%$. Additionally, $80 \times 2000$ $\mathrm{nm}$ showed similar recovery percentages to $80 \times 180 \mathrm{~nm}$ and $80 \times 320 \mathrm{~nm}$ particles. Most surprising, however, was that essentially all particles were recovered in the case of $2 \mathrm{wt} \% 80$ $\times 5000 \mathrm{~nm}$ particles. In contrast to this finding, the lowest filtration efficiency was also associated with $80 \times 5000 \mathrm{~nm}$ particles, specifically those containing $96 \mathrm{wt} \%$ PEGDA, which showed $29 \%$ recovery. This composition and aspect ratio also resulted in clogging of the membrane prior to complete recovery of the solution, while all other particle solutions were collected in full following filtration.

The results obtained from the filtration assays may be rationalized in several ways. First, nanoparticles with smaller aspect ratios, such as $80 \times 180 \mathrm{~nm}$ and $80 \times 320 \mathrm{~nm}$ particles, are essentially small enough to traverse pores on the scale of $0.2 \mu \mathrm{m}$ at all of the PEGDA concentrations studied here without clogging of the pores. Secondly, nanoparticles of all the aspect ratios reported, and having $\leq 10 \mathrm{wt} \%$ PEGDA have significant flexibility and deformability as noted by the results obtained by AFM and persistence length measurements. Together, these factors provide a reasonable explanation for the high filtration efficiencies observed, as longer nanoparticles may be able to enter a pore at any 
point along their axis, bend under the pressures exerted during filtration, and deform to allow the particles to translate through the pores under the same pressures. This scenario is also plausible if the DLS results of the low cross-linked nanoparticles are accounted for, where $D_{\mathrm{h}}$ fell in the range of $200-400 \mathrm{~nm}$. Finally, based on the low deformability of $96 \mathrm{wt}$ $\%$ PEGDA nanoparticles and the large $L_{\mathrm{P}}$ and $D_{\mathrm{h}}$ values determined for $96 \mathrm{wt} \%$ PEGDA 80 $\times 5000 \mathrm{~nm}$ particles, it is not at all surprising that filtration of these particles resulted in a low $\%$ recovery and clogging of the filtration membrane.

Particle solutions were also analyzed by DLS to compare $D_{\mathrm{h}}$ values before and after filtration, looking for any indication of particle scission or breakage. In most cases the particles remained the same size before and after filtration or decreased slightly in size (see Supporting Information), possibly due to breaking up of small particle aggregates during the filtration process. In a few instances particle sizes were found to be larger following filtration, suggesting potential aggregation. It was found that a brief period ( $\sim 30 \mathrm{~min})$ of bath sonication was adequate for breaking up aggregates, as a decrease in $D_{\mathrm{h}}$ was observed, and the post-sonication $D_{\mathrm{h}}$ values were closer to the prefiltration values. Further analysis by SEM showed that $96 \mathrm{wt} \%$ PEGDA particles of all four aspect ratios retained their structural integrity following filtration and also showed less particle aggregation when compared to pre-filtered solutions, corroborating the results found by DLS (see Supporting Information).

\section{Conclusions}

In this report we have demonstrated the ability to fabricate monodisperse filamentous nanoparticles with controlled aspect ratios and deformability using the PRINT technique. As shown using DLS, small nanoparticles (AR $\leq 4$ ) displayed $D_{\mathrm{h}}$ values that were independent of both PEGDA concentration and temperature, whereas the $D_{\mathrm{h}}$ associated with longer nanoparticles ( $A R 225$ ) displayed a dependence on both variables. Additionally, it was found that $D_{\mathrm{h}}$ values of all nanoparticles containing $\leq 10 \mathrm{wt} \%$ PEGDA were similar in value. AFM measurements allowed for a qualitative analysis of particle deformability as a function of PEGDA concentration, which showed a flattening effect as PEGDA content was decreased. The effect of PEGDA concentration was also observed by measuring 2D particle persistence lengths, which showed increased particle rigidity and $L_{\mathrm{P}}$ as a result high crosslinking density. The development of a simple filtration assay aided in determining the efficiency of particle translocation across a porous membrane. With the exception of $80 \times$ $5000 \mathrm{~nm}$ particles containing $96 \mathrm{wt} \%$ PEGDA, all particle sizes and compositions were recovered with $\geq 73 \%$ efficiency.

The ability to modulate physical properties such as hydrodynamic size, deformability, and flexibility make filamentous particles an attractive choice for state-of-the-art nanomedicine applications. Additionally, the ability to effectively filter filamentous nanoparticles will aid in the sterilization of future particle formulations. Future studies with PEG-based hydrogel filamentous nanoparticles will involve correlating aspect ratio and modulus to in vivo characteristics such as biodistribution profiles, tumor uptake, and circulation times.

\section{Supplementary Material}

Refer to Web version on PubMed Central for supplementary material.

\section{Acknowledgments}

We thank Dr. Kevin Herlihy and Dr. Yapei Wang for useful discussions regarding PRINT particle fabrication, Dr. Ashish Pandya for the synthesis of HP4A monomer, and Dr. Ashley Galloway of Liquidia Technologies for helpful conversations regarding filtration procedures. This work was supported by Liquidia Technologies, the National Institutes of Health (R21H2092814) and NIH Pioneer Award (1DP1OD006432), the NIH National Cancer Institute 
- Carolina Center for Nanotechnology Excellence (U54CA119343 \& U54CA151652), and the NSF EAGER Award (DMR-0923604).

\section{References}

1. Tao L, Hu W, Liu Y, Huang G, Sumer BD, Gao J. Shape-specific polymeric nanomedicine: emerging opportunities and challenges. Exp Biol Med. 2011; 236:20-29.

2. Doshi N, Mitragotri S. Macrophages Recognize Size and Shape of Their Targets. PLoS ONE. 2010; 5:e10051. [PubMed: 20386614]

3. Gratton SEA, Ropp PA, Pohlhaus PD, Luft JC, Madden VJ, Napier ME, DeSimone JM. The effect of particle design on cellular internalization pathways. Proc Natl Acad Sci USA. 2008; 105:1161311618. [PubMed: 18697944]

4. Gratton SEA, Williams SS, Napier ME, Pohlhaus PD, Zhou Z, Wiles KB, Maynor BW, Shen C, Olafsen T, Samulski ET, DeSimone JM. The Pursuit of a Scalable Nanofabrication Platform for Use in Material and Life Science Applications. Acc Chem Res. 2008; 41:1685-1695. [PubMed: 18720952]

5. Euliss LE, DuPont JA, Gratton S, DeSimone J. Imparting size, shape, and composition control of materials for nanomedicine. Chem Soc Rev. 2006; 35:1095-1104. [PubMed: 17057838]

6. Champion JA, Mitragotri S. Role of target geometry in phagocytosis. Proc Natl Acad Sci USA. 2006; 103:4930-4934. [PubMed: 16549762]

7. Hendrickson GR, Lyon LA. Microgel Translocation through Pores under Confinement. Angew Chem Int Ed. 2010; 49:2193-2197.

8. Banquy X, Suarez F, Argaw A, Rabanel JM, Grutter P, Bouchard JF, Hildgen P, Giasson S. Effect of mechanical properties of hydrogel nanoparticles on macrophage cell uptake. Soft Matter. 2009; 5:3984-3991.

9. Haghgooie R, Toner M, Doyle PS. Squishy Non-Spherical Hydrogel Microparticles. Macromol Rapid Comm. 2009; 31:128-134.

10. Merkel TJ, Jones SW, Herlihy KP, Kersey FR, Shields AR, Napier M, Luft JC, Wu H, Zamboni WC, Wang AZ, Bear JE, DeSimone JM. Using mechanobiological mimicry of red blood cells to extend circulation times of hydrogel microparticles. Proc Natl Acad Sci USA. 2011; 108:586-591. [PubMed: 21220299]

11. Geisbert TW, Jahrling PB. Exotic emerging viral diseases: progress and challenges. Nat Med. 2004; 10:S110-S121. [PubMed: 15577929]

12. Young KD. Bacterial morphology: why have different shapes? Curr Opin Microbiol. 2007; 10:596-600. [PubMed: 17981076]

13. Christian DA, Cai S, Garbuzenko OB, Harada T, Zajac AL, Minko T, Discher DE. Flexible Filaments for in Vivo Imaging and Delivery: Persistent Circulation of Filomicelles Opens the Dosage Window for Sustained Tumor Shrinkage. Mol Pharm. 2009; 6:1343-1352. [PubMed: 19249859]

14. Simone EA, Dziubla TD, Discher DE, Muzykantov VR. Filamentous Polymer Nanocarriers of Tunable Stiffness that Encapsulate the Therapeutic Enzyme Catalase. Biomacromolecules. 2009; 10:1324-1330. [PubMed: 19385657]

15. Geng Y, Dalhaimer P, Cai S, Tsai R, Tewari M, Minko T, Discher DE. Shape effects of filaments versus spherical particles in flow and drug delivery. Nat Nanotechnol. 2007; 2:249-255. [PubMed: 18654271]

16. Younghoon K, Dalhaimer P, Christian DA, Discher DE. Polymeric worm micelles as nano-carriers for drug delivery. Nanotechnology. 2005; 16:S484. [PubMed: 21727469]

17. Sharma G, Valenta DT, Altman Y, Harvey S, Xie H, Mitragotri S, Smith JW. Polymer particle shape independently influences binding and internalization by macrophages. J Control Release. 2010; 147:408-412. [PubMed: 20691741]

18. Champion J, Mitragotri S. Shape Induced Inhibition of Phagocytosis of Polymer Particles. Pharm Res. 2009; 26:244-249. [PubMed: 18548338]

19. Lu Z, Qiao Y, Zheng XT, Chan-Park MB, Li CM. Effect of particle shape on phagocytosis of CdTe quantum dot-cystine composites. Med Chem Comm. 2010; 1:84-86. 
20. Bauer LA, Birenbaum NS, Meyer GJ. Biological applications of high aspect ratio nanoparticles. J Mater Chem. 2004; 14:517-526.

21. Liu Z, Cai W, He L, Nakayama N, Chen K, Sun X, Chen X, Dai H. In vivo biodistribution and highly efficient tumour targeting of carbon nanotubes in mice. Nat Nanotechnol. 2007; 2:47-52. [PubMed: 18654207]

22. Arnida, Janát-Amsbury MM, Ray A, Peterson CM, Ghandehari H. Geometry and surface characteristics of gold nanoparticles influence their biodistribution and uptake by macrophages. Eur J Pharm Biopharm. 2011; 77:417-423. [PubMed: 21093587]

23. Won YY, Davis HT, Bates FS. Giant Wormlike Rubber Micelles. Science. 1999; 283:960-963. [PubMed: 9974383]

24. Discher DE, Eisenberg A. Polymer Vesicles. Science. 2002; 297:967-973. [PubMed: 12169723]

25. Geng Y, Discher DE. Hydrolytic Degradation of Poly(ethylene oxide)-block-Polycaprolactone Worm Micelles. J Am Chem Soc. 2005; 127:12780-12781. [PubMed: 16159254]

26. Geng Y, Discher DE. Visualization of degradable worm micelle breakdown in relation to drug release. Polymer. 2006; 47:2519-2525.

27. Dalhaimer P, Bermudez H, Discher DE. Biopolymer mimicry with polymeric wormlike micelles: Molecular weight scaled flexibility, locked-in curvature, and coexisting microphases. J Polym Sci B Pol Phys. 2004; 42:168-176.

28. Dalhaimer P, Bates FS, Discher DE. Single Molecule Visualization of Stable, Stiffness-Tunable, Flow-Conforming Worm Micelles. Macromolecules. 2003; 36:6873-6877.

29. Ho CC, Keller A, Odell JA, Ottewill RH. Preparation of monodisperse ellipsoidal polystyrene particles. Colloid Polym Sci. 1993; 271:469-479.

30. Kolhar P, Doshi N, Mitragotri S. Polymer Nanoneedle-Mediated Intracellular Drug Delivery. Small. 2011; 7:2094-2100. [PubMed: 21695782]

31. Tao L, Zhao XM, Gao JM, Hu W. Lithographically defined uniform worm-shaped polymeric nanoparticles. Nanotechnology. 2010; 21:095301. [PubMed: 20110578]

32. Buyukserin F, Aryal M, Gao J, Hu W. Fabrication of Polymeric Nanorods Using Bilayer Nanoimprint Lithography. Small. 2009; 5:1632-1636. [PubMed: 19347857]

33. Rolland JP, Maynor BW, Euliss LE, Exner AE, Denison GM, DeSimone JM. Direct Fabrication and Harvesting of Monodisperse, Shape-Specific Nanobiomaterials. J Am Chem Soc. 2005; 127:10096-10100. [PubMed: 16011375]

34. Gratton SEA, Pohlhaus PD, Lee J, Guo J, Cho MJ, DeSimone JM. Nanofabricated particles for engineered drug therapies: A preliminary biodistribution study of PRINT(TM) nanoparticles. J Control Release. 2007; 121:10-18. [PubMed: 17643544]

35. Canelas DA, Herlihy KP, DeSimone JM. Top-down particle fabrication: control of size and shape for diagnostic imaging and drug delivery. Wiley Interdiscip Rev Nanomed Nanobiotechnol. 2009; 1:391-404. [PubMed: 20049805]

36. Petros RA, Ropp PA, DeSimone JM. Reductively Labile PRINT Particles for the Delivery of Doxorubicin to HeLa Cells. J Am Chem Soc. 2008; 130:5008-5009. [PubMed: 18355010]

37. Kelly JY, DeSimone JM. Shape-Specific, Monodisperse Nano-Molding of Protein Particles. J Am Chem Soc. 2008; 130:5438-5439. [PubMed: 18376832]

38. Enlow EM, Luft JC, Napier ME, DeSimone JM. Potent Engineered PLGA Nanoparticles by Virtue of Exceptionally High Chemotherapeutic Loadings. Nano Lett. 2011; 11:808-813. [PubMed: 21265552]

39. McAllister K, Sazani P, Adam M, Cho MJ, Rubinstein M, Samulski RJ, DeSimone JM. Polymeric Nanogels Produced via Inverse Microemulsion Polymerization as Potential Gene and Antisense Delivery Agents. J Am Chem Soc. 2002; 124:15198-15207. [PubMed: 12487595]

40. Guzmán J, Iglesias MT, Riande E, Compañ V, Andrio A. Synthesis and polymerization of acrylic monomers with hydrophilic long side groups. Oxygen transport through water swollen membranes prepared from these polymers. Polymer. 1997; 38:5227-5232.

41. Roiter, Y.; Minko, S. 2D Single Molecules, v.1.0.3.5. Freeware

42. von Berlepsch H, Harnau L, Reineker P. Persistence Length of Wormlike Micelles from Dynamic Light Scattering. J Phys Chem B. 1998; 102:7518-7522. 
43. Maguire JF. Rotational dynamics of rigid rods. A dynamic light-scattering study. J Chem Soc, Faraday Trans 2. 1981; 77:513-518.

44. Kabanov AV, Vinogradov SV. Nanogels as Pharmaceutical Carriers: Finite Networks of Infinite Capabilities. Angew Chem Int Ed. 2009; 48:5418-5429.

45. Flory, PJ. Principles of Polymer Chemistry. Cornell University Press; Ithaca, NY: 1953.

46. Cruise GM, Scharp DS, Hubbell JA. Characterization of permeability and network structure of interfacially photopolymerized poly(ethylene glycol) diacrylate hydrogels. Biomaterials. 1998; 19:1287-1294. [PubMed: 9720892]

47. Lin CC, Anseth K. PEG Hydrogels for the Controlled Release of Biomolecules in Regenerative Medicine. Pharm Res. 2009; 26:631-643. [PubMed: 19089601]

48. Iza M, Stoianovici G, Viora L, Grossiord JL, Couarraze G. Hydrogels of poly(ethylene glycol): mechanical characterization and release of a model drug. J Control Release. 1998; 52:41-51. [PubMed: 9685934]

49. Chan V, Zorlutuna P, Jeong JH, Kong H, Bashir R. Three-dimensional photopatterning of hydrogels using stereolithography for long-term cell encapsulation. Lab Chip. 2010; 10:20622070. [PubMed: 20603661]

50. Landau, LD.; Lifschitz, EM. Statistical Physics. 3rd. Pergamon Press; Oxford, New York: 1980.

51. Maier B, Rädler JO. Conformation and Self-Diffusion of Single DNA Molecules Confined to Two Dimensions. Phys Rev Lett. 1999; 82:1911-1914.

52. Gittes F, Mickey B, Nettleton J, Howard J. Flexural rigidity of microtubules and actin filaments measured from thermal fluctuations in shape. J Cell Biol. 1993; 120:923-934. [PubMed: 8432732]

53. Ott A, Magnasco M, Simon A, Libchaber A. Measurement of the persistence length of polymerized actin using fluorescence microscopy. Phys Rev E. 1993; 48:R1642-R1645.

54. Dalhaimer P, Wagner OI, Leterrier JF, Janmey PA, Aranda-Espinoza H, Discher DE. Flexibility transitions and looped adsorption of wormlike chains. J Polym Sci B Pol Phys. 2005; 43:280-286.

55. Mücke N, Kreplak L, Kirmse R, Wedig T, Herrmann H, Aebi U, Langowski J. Assessing the Flexibility of Intermediate Filaments by Atomic Force Microscopy. J Mol Biol. 2004; 335:12411250. [PubMed: 14729340]

56. Rivetti C, Guthold M, Bustamante C. Scanning Force Microscopy of DNA Deposited onto Mica: Equilibration versus Kinetic Trapping Studied by Statistical Polymer Chain Analysis. J Mol Biol. 1996; 264:919-932. [PubMed: 9000621]

57. Trachtenberg, S.; Hammel, I. Determining the persistence length of biopolymers and rod-like macromolecular assemblies from electron microscope images and deriving some of their mechanical properties. In: Mendez-Vilas, A.; Diaz, J., editors. Microscopy: Science, Technology, Applications and Education. Vol. 3. Formatex Research Center; 2010. p. 1690-1695.

58. Whitehead TA, Je E, Clark DS. Rational shape engineering of the filamentous protein $\gamma$ prefoldin through incremental gene truncation. Biopolymers. 2009; 91:496-503. [PubMed: 19189379]

59. Hobbs SK, Monsky WL, Yuan F, Roberts WG, Griffith L, Torchilin VP, Jain RK. Regulation of transport pathways in tumor vessels: Role of tumor type and microenvironment. Proc Natl Acad Sci USA. 1998; 95:4607-4612. [PubMed: 9539785]

60. Hall JB, Dobrovolskaia MA, Patri AK, McNeil SE. Characterization of nanoparticles for therapeutics. Nanomedicine. 2007; 2:789-803. [PubMed: 18095846]

61. Markovsky E, Koroukhov N, Golomb G. Additive-free albumin nanoparticles of alendronate for attenuating inflammation through monocyte inhibition. Nanomedicine. 2007; 2:545-553.

[PubMed: 17716137] 


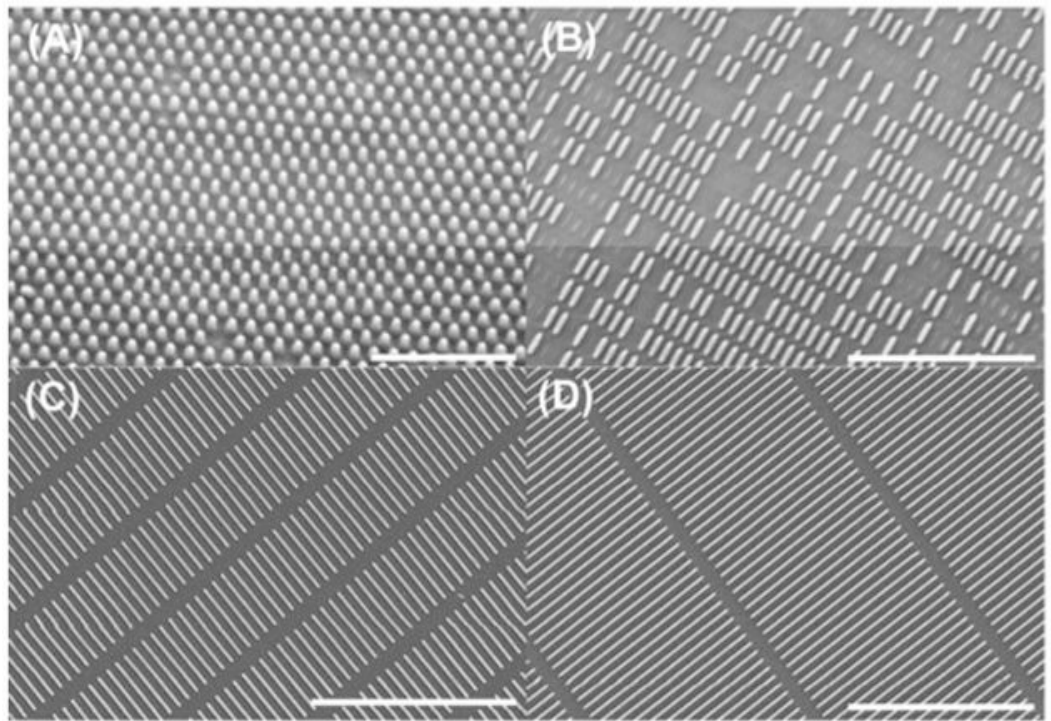

Figure 1.

Representative SEM images of the PEG-based hydrogel $80 \mathrm{~nm}$ particle series. Lengths for the particles shown are (A) $180 \mathrm{~nm}$, (B) $320 \mathrm{~nm}$, (C) $2000 \mathrm{~nm}$, and (D) $5000 \mathrm{~nm}$. Scale bar values are (A) $1 \mu \mathrm{m}$, (B) $3 \mu \mathrm{m}$, and (C) \& (D) $5 \mu \mathrm{m}$. 


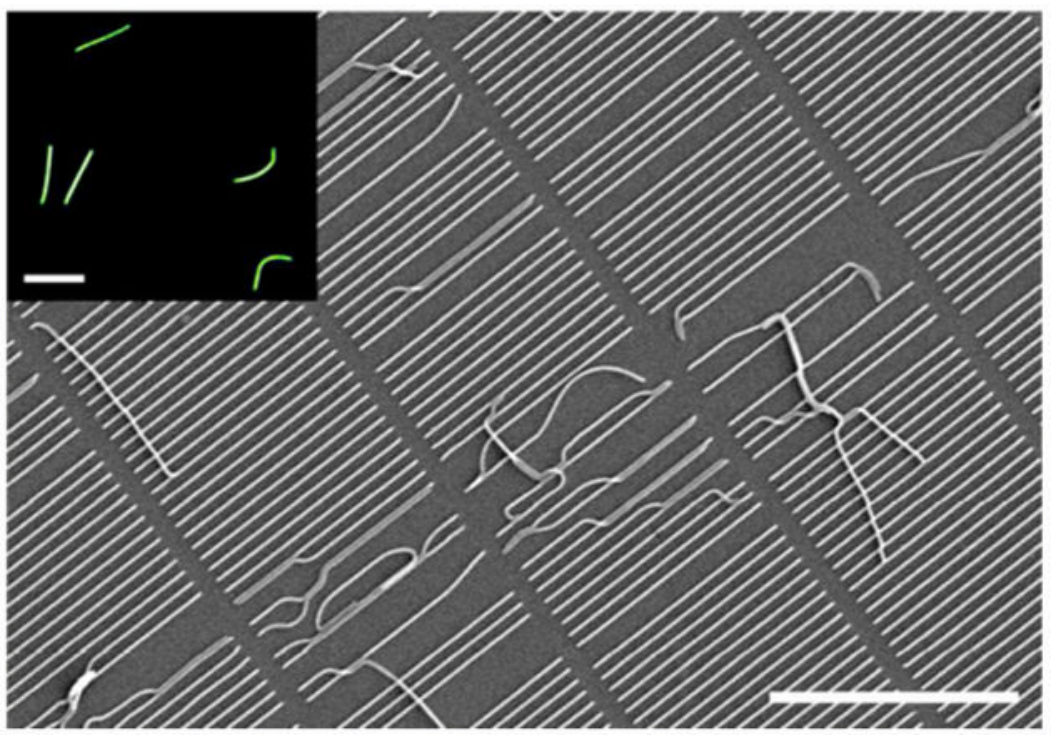

Figure 2.

A representative SEM image showing $96 \mathrm{wt} \%$ PEGDA $80 \times 5000 \mathrm{~nm}$ particles. Several particles have detached from the PET sheet and display some flexibility. Inset: a fluorescence microscope image of the harvested particles shown in the SEM image. Scale bars for both images are $5 \mu \mathrm{m}$. 

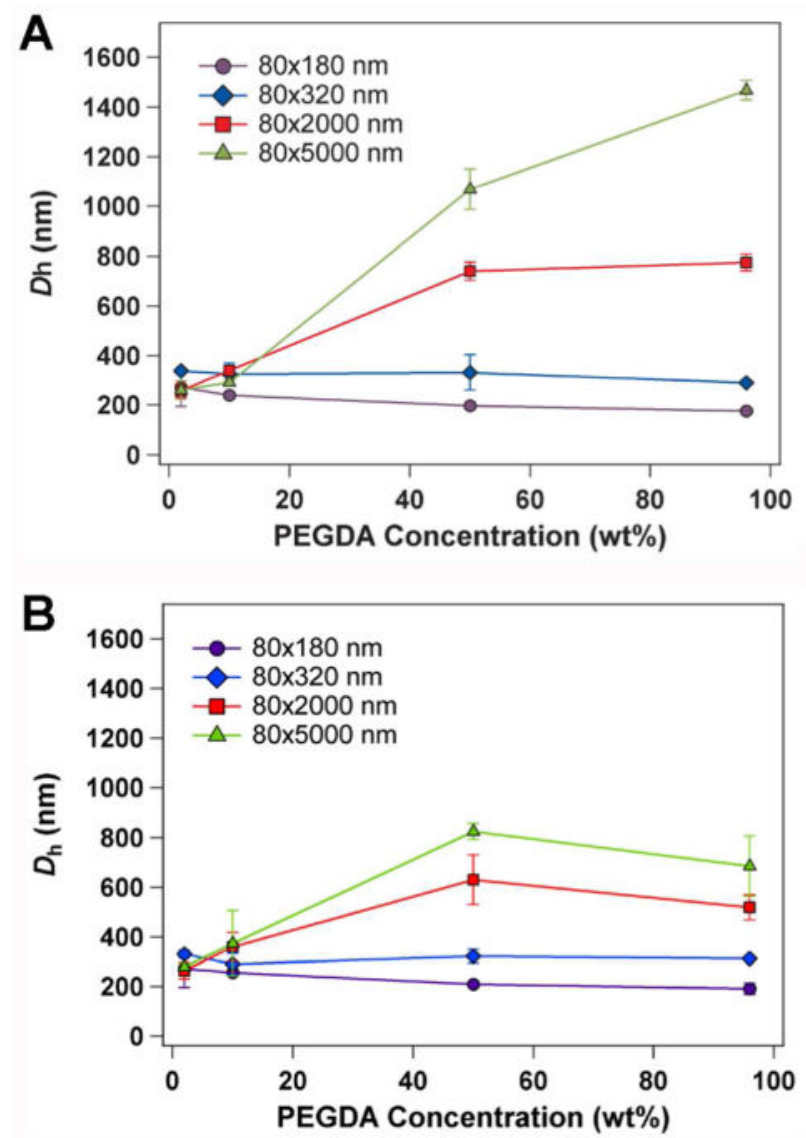

Figure 3.

Effective hydrodynamic diameter $\left(D_{\mathrm{h}}\right)$ data for $80 \mathrm{~nm}$ particle series at (A) $25^{\circ} \mathrm{C}$ and (B) 37 ${ }^{\circ} \mathrm{C}$. DLS results show particle size is a function of temperature and PEGDA concentration. 
A

Figure 4.

Representative 3D AFM height images of 2 wt $\%$ PEGDA (A) and 96 wt $\%$ PEGDA (B) $80 \times$ $180 \mathrm{~nm}$ particles showing deformability as a function of cross-linker concentration. 

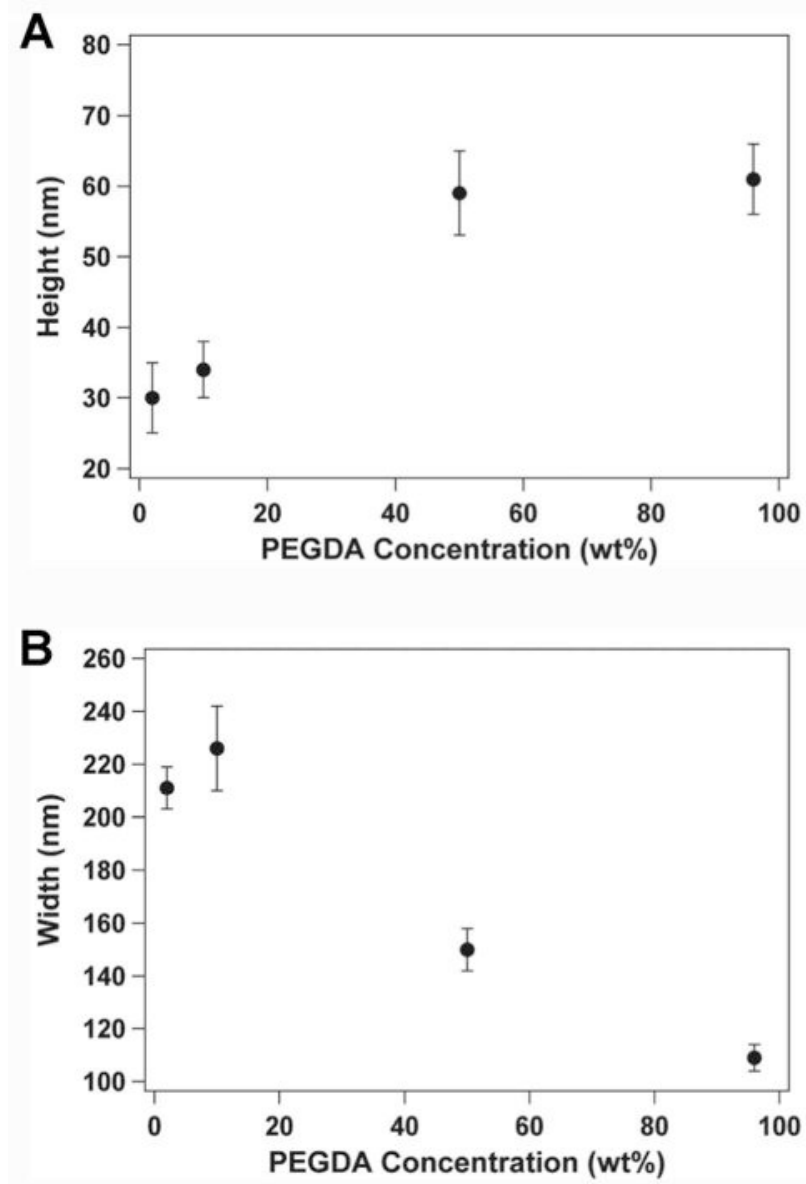

Figure 5.

AFM analysis of particle deformability for $80 \times 180 \mathrm{~nm}$ particles as a function of PEGDA concentration. Nanoparticle height (A) and width (B) are observed to increase and decrease, respectively, with increasing PEGDA concentration. 


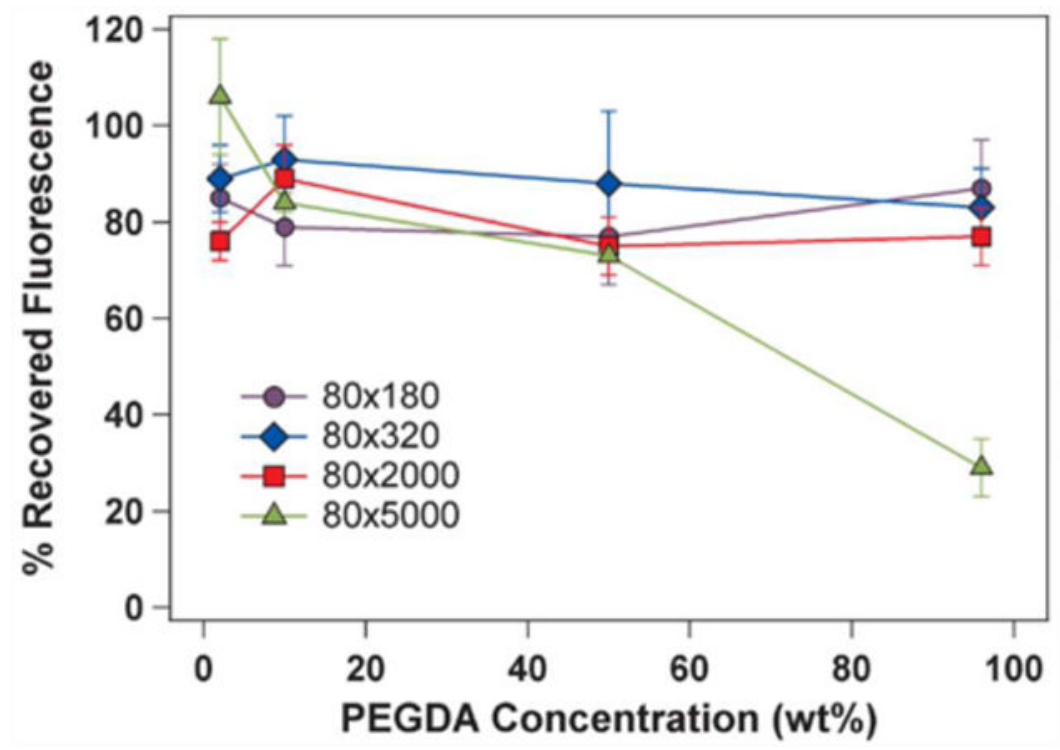

Figure 6.

Percent recovered fluorescence for the $80 \mathrm{~nm}$ particle series as a function of PEGDA concentration. These percentages also refer to the percentage of particles recovered following filtration. 
Table 1

Characteristic length parameters determined from 2D SEM images of $80 \times 5000 \mathrm{~nm}$ nanoparticles

\begin{tabular}{ccc}
\hline PEGDA $(\mathbf{w t} \%)$ & $\boldsymbol{L}_{\mathbf{C}}(\boldsymbol{\mu} \mathbf{m})$ & $\boldsymbol{L}_{\mathbf{P}}(\boldsymbol{\mu} \mathbf{m})$ \\
\hline 10 & $5.40 \pm 0.37$ & 1.61 \\
50 & $5.34 \pm 0.55$ & 1.57 \\
96 & $4.97 \pm 0.10$ & 16.96 \\
\hline
\end{tabular}

